Article

\title{
Practical Demonstration of 5G NR Transport Over-Fiber System with Convolutional Neural Network
}

\author{
Muhammad Usman Hadi (D)
}

Citation: Hadi, M.U. Practical Demonstration of 5G NR Transport Over-Fiber System with Convolutional Neural Network. Telecom 2022, 3, 103-117. https:// doi.org/10.3390/telecom3010006

Academic Editors: Mohammad Dehghani Soltani and

Iman Tavakkolnia

Received: 28 December 2021

Accepted: 27 January 2022

Published: 2 February 2022

Publisher's Note: MDPI stays neutral with regard to jurisdictional claims in published maps and institutional affiliations.

Copyright: (c) 2022 by the author. Licensee MDPI, Basel, Switzerland. This article is an open access article distributed under the terms and conditions of the Creative Commons Attribution (CC BY) license (https:// creativecommons.org/licenses/by/ $4.0 /)$.
School of Engineering, Ulster University, Newtownabbey BT37 0QB, UK; usmanhadi@ieee.org or m.hadi@ulster.ac.uk

\begin{abstract}
This study describes an experimental realization using digital predistortion (DPD) for a fifth generation (5G) multiband new radio (NR) optical front haul (OFH) based Radio over Fiber (RoF) link. For the performance enhancement and complexity reduction of RoF links, a novel Convolutional Neural Network (CNN) based DPD technique is proposed, followed by comparisons with the generalised memory polynomial (GMP) based DPD method. To support enhanced mobile broad band scenario, the experimental testbed uses the $5 \mathrm{G}$ NR waveforms at $10 \mathrm{GHz}$ with $20 \mathrm{MHz}$ bandwidth and a flexible-waveform signal at $3 \mathrm{GHz}$ with $20 \mathrm{MHz}$ bandwidth. For $10 \mathrm{~km}$ of typical single mode fiber, a Mach Zehnder Modulator with two distinct radio frequency waveforms modulates a $1310 \mathrm{~nm}$ optical carrier utilizing distributed feedback laser. The error vector magnitude and number of estimated coefficients, and multiplications are all used to describe the experimental outcomes. The goal of the research is to see if CNN-based DPD improves performance while lowering complexity levels to meet 3GPP Release 17 criteria.
\end{abstract}

Keywords: digital predistortion; convolutional neural network; radio over fiber; convolutional neural network; error vector magnitude

\section{Introduction}

With major developments in the fifth generation known as $5 \mathrm{G}$ and beyond $5 \mathrm{G}$, the centralization of radio access network (RAN) has been established due to the ever increasing rate of base stations (BS) [1], which decreases capital expenditure by simplifying network management [2]. An optical fronthaul (OFH/FH) simply connects the base band units $(B B U)$ to remote radio heads $(R R H)$ to facilitate C-RAN (see Figure 1). Due to unlimited benefits and increase in wireless link reach for all kinds of applications such as short to long link applications, microwave photonics-based solutions such as Radio over Fiber (RoF) have a higher significance connecting the BBUs with RRUs [1,2] with 5G in the operational stage in most parts of the world [1,2].

Various RoF variants have been proposed in recent years, including Analog Radio over Fiber (A-RoF) [1-5], Digital transport over Fiber (D-RoF) [6-9], Sigma Delta transport/ Radio over Fiber (SD-RoF) [10-12], and others (see Figure 2). A-RoF links are, to some extent, the simplest and most cost-effective option; nonetheless, they suffer from nonlinearities generated from signal impairments and components such as laser modules, fiber, and photodiodes. Using D-RoF or SD-RoF is one of the alternative options.

When it comes to D-RoF systems, the analogue to digital (ADC) and digital to analogue (DAC) required make the process exceedingly costly. Furthermore, common public radio interface (CPRI) restrictions exist due to high data rate capacity and limited bandwidth. The SD-RoF can be used to bypass the CPRI bottleneck. ADCs and DACs aren't needed because the method is based on sigma-delta modulation, which only uses one bit of ADC. However, the method is difficult, thus it's not recommended. Furthermore, because the quantization noise is significant for $1 \mathrm{bit}$, an extra band pass filter (BPF) at the RRH is required. However, this added complexity is not the only issue that needs to be addressed for S-DRoF implementation; the addition of BPF introduces additional noise in amplitude and phase, which necessitates a second method to remove these nonlinearities [13]. 
Optical Fronthaul (OFH)

Remote Radio Head (RRH)

Pico Cell Range 3 GHz

(Q)

Optical Backhaul

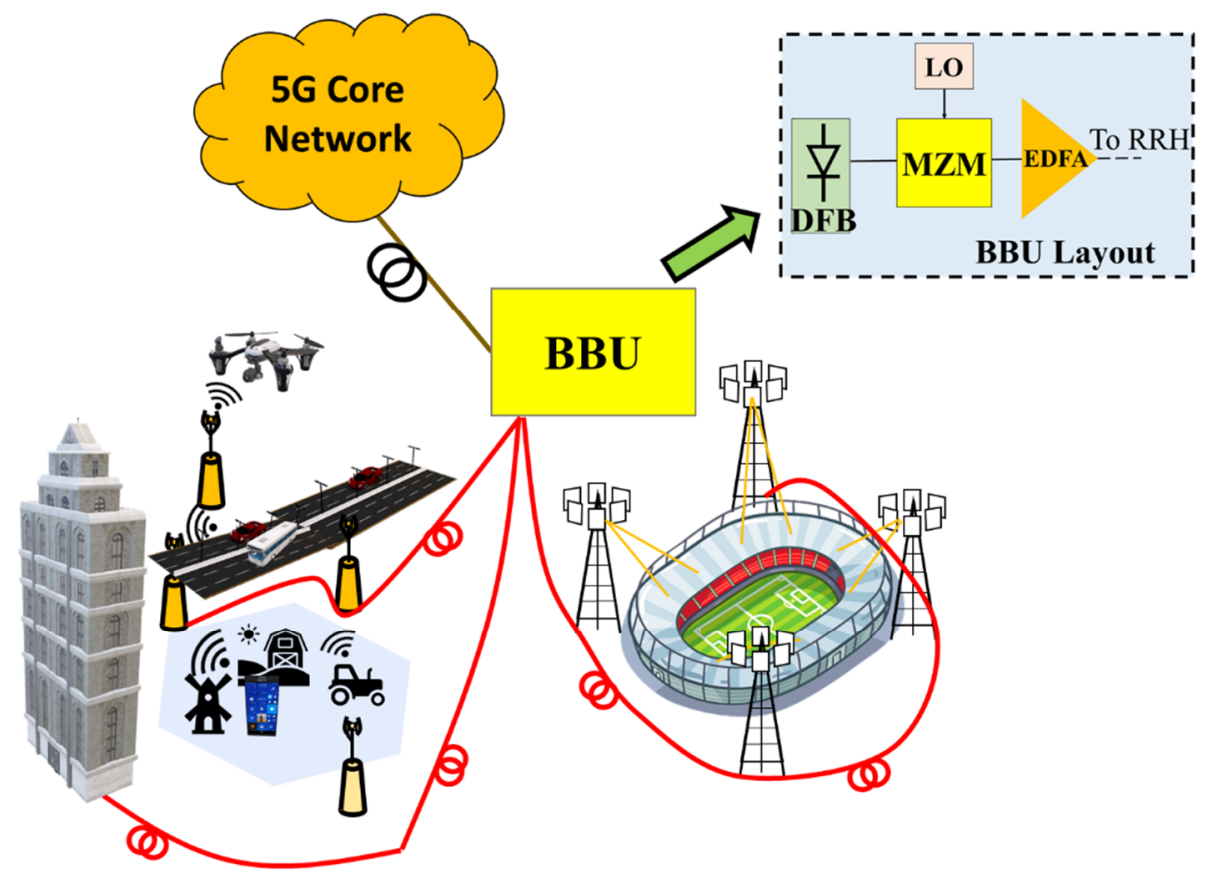

Figure 1. Schema of RAN 5G covering the back and fronthaul with application scenarios such as houses, sports fields and transportation, etc.

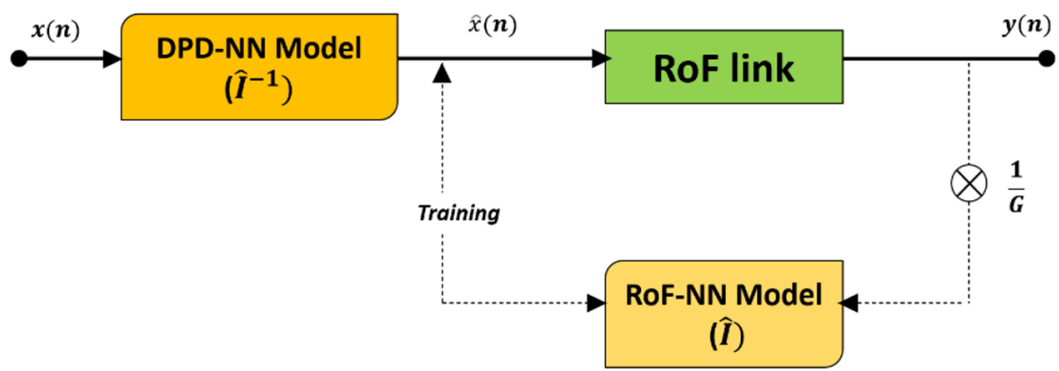

Figure 2. RoF system block diagram with Radio over Fiber and CNN based DPD system. Transmission of $\mathrm{I} / \mathrm{P}$ and $\mathrm{O} / \mathrm{P}$ across the general RoF link yields the RoF NN model $\hat{I}$. After that, we backpropagate error through $I$ to train $I^{-1}$. The DPD-RoF model is then linearized by linking it to an RoF link. DPD is done in the digital baseband, which eliminates the need for DACs and ADCs The link that how CNN is made and trained is shown in Algorithm 1.

It is clear that utilising alternative techniques (D-RoF/SD-RoF) is time, resource and power consuming. As a result, the A-RoF systems are the preferable alternative for optical fronthaul due to their legacy, infrastructure, and cost-effectiveness (OFH). Mitigating nonlinearities in RoF transmission is critical for maximising the system's potential and has become worthy of discussion. To address the widespread challenges in all of these distinct fields, a variety of strategies have been employed. The ones widely used are mentioned in Section 2 under the literature review.

As transmission quality diminishes, the laser nonlinearities become more significant. When it comes to long-range networks, however, nonlinearities caused by a combination of fiber dispersion and laser chirp are frequently the primary cause of impairments and degradation [7]. Due to the high peak-to-average power ratio (PAPR), Orthogonal Frequency Division Modulated (OFDM) signals, such as the highlighted 5G transmission, are also susceptible to similar distortions, i.e., PAPR.

This study introduces nonlinear behavior and signal degradation compensation for OFH based RoF systems using 5G NR based RoF technology, to the best of the authors' 
knowledge. The novelty of this study is numerous, following a thorough literature assessment on nonlinearity mitigation in Section 2 :

1. In the experimental testbed, multiband 5G NR signals are used to cover enhanced mobile wide band (eMBB) scenarios and small cells for $3 \mathrm{GHz}$ and $10 \mathrm{GHz}$, respectively.

2. An abled DPD method based on a Convolutional Neural Network (CNN) is proposed and demonstrated. In comparison to existing learning architectures, the proposed DPD identification approach has a better performance and lower complexity than our previous machine learning approach.

3. Finally, a simple CNN-based DPD algorithm is proposed as an upgrade to our previously published DPD-based technique based on deep learning for $20 \mathrm{MHz}$ with 5G New Radio (NR) based RoF links. A new sort of training is used to implement the CNN DPD technique, which does not use In-direct Learning Architecture (ILA). We first use an RoF CNN to simulate the generic RoF connection and then use this to train the proposed DPD CNN by backpropagating the mistakes.

4. A comparative experimental investigation was conducted in which the previously proposed ILA-based GMP method was benchmarked against the CNN technique and compared utilising a 5G NR multiband signal. Error Vector Magnitude (EVM) and multiplications and coefficients required measuring complexities are used to evaluate the performance.

\section{Literature Review}

In this section, only Machine Learning Methods are used to discuss the linearization methodologies that have been inferred for the RoF systems. Linearization of OFH has been a significant research area as summarized below in Table 1 . There has always been a drive to have better linearization to get higher performance that has shifted the focus towards Machine Learning (ML). ML being the new avenue is the core discussion of this work so a recent literature review has been discussed for all methods with specific importance to machine learning methods. For the reduction of the RoF system impairments, a thorough literature review is described in Table 1. The table outlines the method used, the type of linearization used, the category, the parameters examined, and the benefits and drawbacks of each method. 
Table 1. Nonlinearity mitigation methods employed $(\mathrm{Dig}=$ Digital, Elec $=$ Electrical, Opt $=$ Optical, $\mathrm{ML}=$ Machine Learning, $\mathrm{ACLR}=\mathrm{Adjacent}$ Channel Leakage Ratio, EVM = Error Vector Magnitude are used to abbreviate in the table).

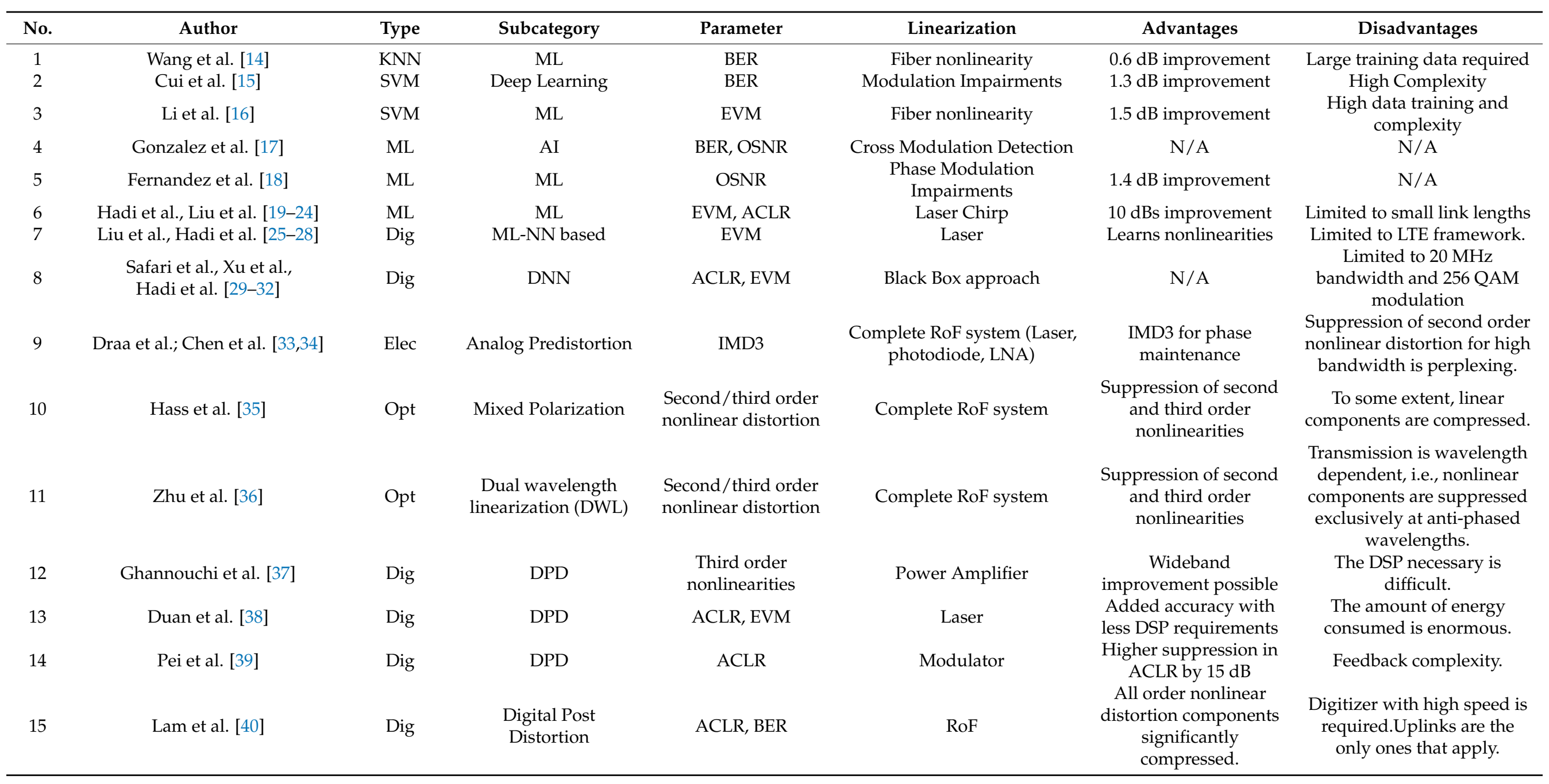


Table 1. Cont.

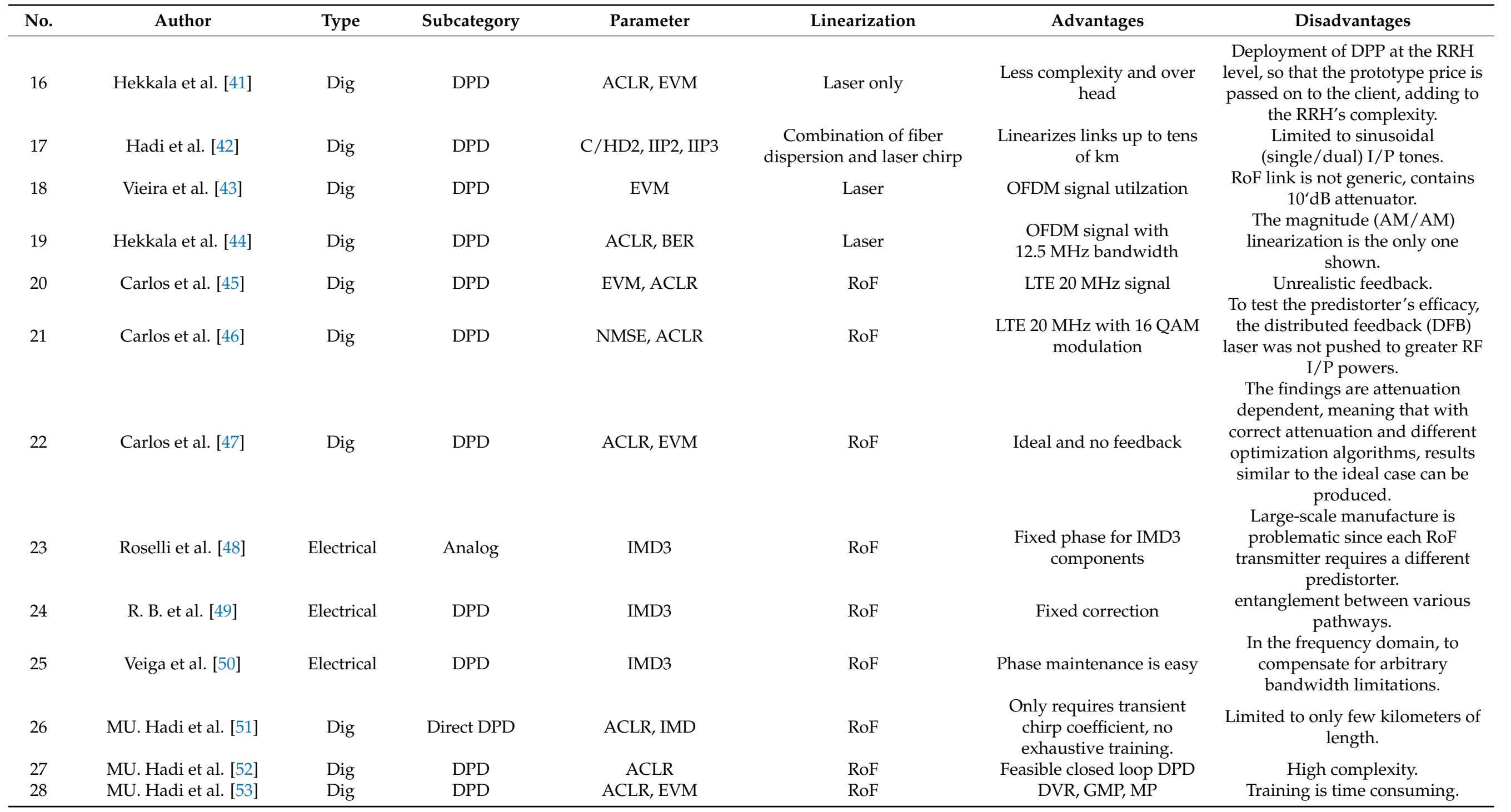




\section{Convolutional Neural Network Architecture}

Convolution Neural Networks (CNN) are advanced networks that, like the recommended NN-based DPD model, require a large amount of training data. The RoF link is then cascaded with this model, but the outcome is unknown. However, because the output of an RoF connection is known, we create an RoF NN model and train it to mimic the original RoF link. We can now backpropagate through this RoF CNN and adjust the parameters in the recommended CNN DPD model after we have formulated it.

The convolutional layer is the first layer of a CNN. With the help of a kernel, it extracts features from input data and outputs a feature map (convoluted data).

The kernel or filter (kernel matrix), input data (input matrix), and feature map are all components of a convolutional operation.

Assuming the examined RoF link has $H(n)$ function and $y(n)$ as an output signal, and that a baseband signal $x(n)$ must be delivered over it where $H(n)=\frac{y(n)}{x(n)}$, DPD tries to calculate the inverse transfer function of this RoF link, represented by $\hat{I}^{-1}$, whose output is then indicated by $\hat{x}(n)$.

This can be written as:

$$
\hat{x}(n)=\hat{I}^{-1}(x(n))
$$

while,

$$
y(n)=G x(n)=I(\hat{x}(n))
$$

as $G$ represents the gain. The $C N N$ here finds out the $\hat{I}^{-1}$ utilized for predistortion. A direct training cannot be done for establishing the CNN for DPD as the ideal $\hat{x}(n)$ is not known as illustrated in Figure 2.

Initially, the second CNN simulates the RoF link. Here, $\hat{x}(n)$ is an input and $\frac{y(n)}{G}$ as output for a generic RoF link. This leads to the CNN learning, and it can now identify an approximate transfer function $\hat{I}$. The model weights are fixed once the RoF CNN model is formed, and then it is connected to the CNN DPD model. To calculate error using a loss function, we now use the original input, $x(n)$ and output as training data. We then backpropagate it through $\hat{I}$ to train $\hat{I}^{-1}$.

\subsection{CNN Model Salient Features}

This section will go through all the characteristics of CNN that are required for use in a DPD-based RoF system. The characteristics that make a CNN model are discussed in this section one by one. These characteristics serve as a foundation of the successful implementation of the CNN.

\subsubsection{Optimizer}

The Adam algorithm amalgamates the Momentum and RMS functions, which means that it keeps an exponentially decaying average of prior squared gradients as well as an exponentially decaying average of previous gradients. Due to the component of speed and the ability to adjust gradients, Adam is an effective optimizer. This is also why it is the most commonly utilised optimizer. ADAM has outperformed the competition, particularly in terms of convergence speed, which is why it is the most widely used and efficient optimizer.

\subsubsection{Activation Functions}

A node's capacity is not defined apriori. The activation helps to deduce it. It accomplishes this by establishing a relationship between the node's various weights and biases and then applying the relationship to the node as a function, so generating responses. It also assists it in learning complex data patterns. They convert the node's incoming signals into an output signal that will either be used in the network's next layer or will be the output. In contrast to the gradient of sigmoids, which grows smaller as the absolute value of gradient increases, the chance of a vanishing gradient is lesser when considering ReLu. ReLu has a constant gradient, which aids learning and makes it the better choice. 


\subsubsection{Regularization}

$\mathrm{CNN}$ with high learning parameters and sparse or noisy training data are prone to overfitting problems. Overfitting occurs when a model improves its performance on training data but fails to classify fresh test instances that are part of the same domain problem. We employed an L2 regularization approach to avoid this problem.

In this method, a regularization term is taken into account when updating the cost function. This regularization term reduces the value of the weight matrices by resulting in a simpler model, minimizing the problem of overfitting greatly.

In L2, we have:

$$
\text { Loss }=\text { error }+\lambda \sum_{i=1}^{N}\left|w_{i}^{2}\right|
$$

where $w$ is weight and $\lambda$ is the regularization parameter (which is adjusted for more exact results). The L2 method is known as a decay approach for updating weights because it forces the weights to decay towards zero, though they never reach zero exactly.

We have developed a foundation of all components of a CNN, the DPD CNN, which was used to distort the RoF system, and the replicated RoF CNN, which is needed for training this DPD CNN, based on the conversation so far. The CNN used here is a feedforward fully connected network with $N$ hidden layers and $K$ neurons per hidden layer. Figure 3 depicts the symbolic structure of the employed CNN. Due to the complex nature of baseband signals, which represent both the real and imaginary components of the signal, the $\mathrm{CNN}$ has two inputs and two outputs. At least one of the several hidden levels has been activated using the ReLu function (owing to its lower complexity).

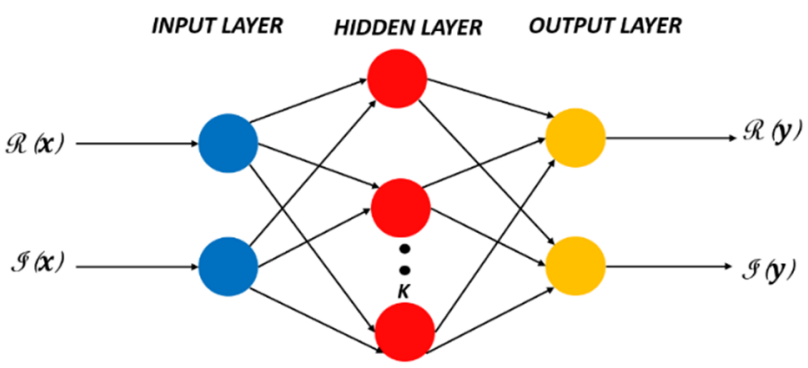

Figure 3. $N$ number of hidden layers has $K$ neurons per hidden layer, as shown in the schematic picture of the feedforward fully connected NN structure utilised.

The $\mathrm{O} / \mathrm{P}$ for the primary layer (hidden) is represented:

$$
l_{1}(n)=f\left(W_{1}\left[\begin{array}{c}
\mathbb{R}(x) \\
\mathbb{I}(x)
\end{array}\right]+b_{1}\right)
$$

where, $l_{1}$ represents the primary hidden output layer, $f$ is the nonlinear function of activation and $W_{1}$ is the weight and $b_{1}$ is the bias for the first output layer in the network.

The general output for the $\mathrm{i}^{\text {th }}$ layer is represented as:

$$
l_{i}(n)=f\left(W_{i} h_{i-1}(n)+b_{i}\right)
$$

where, $i \in \mathbb{N}: 2 \leq i \leq N$.

After $N$ hidden layers, the ultimate result will be:

$$
\hat{x}(n)=W_{N+1} h_{N}+b_{N+1}
$$

\subsubsection{Training Algorithm}

The CNN DPD model was trained using the following algorithm. Mean Square Error (MSE) is employed as the loss measuring function, ADAM is used for the optimization, 
ReLu is used as activation function and backpropagation is used to update the weights. To improve the performance, $\mathrm{Z}$ number of iterations are used.

As previously stated, we use the input and the output of the original RoF connection to train our emulated RoF CNN model, and after this model is acquired, we connect the CNN DPD model to it. Then, once this training is complete, we attach the actual RoF link to this DPD CNN and begin the predistortion process (see pseudocode in Algorithm 1). It is shown in the algorithm that the CNN is made on $I(\hat{x}(n))$ with updates on $\hat{I}$ followed by the pre-distortion functionality usage in terms of $\hat{I}^{-1}(x(n))$ as shown in Figure 2.

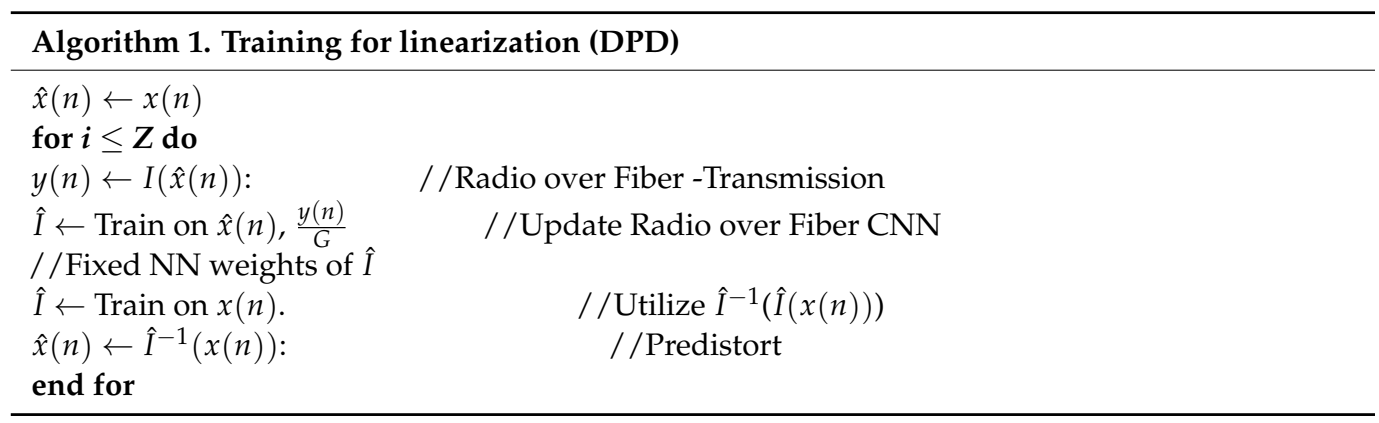

\section{Experimental Setup}

A scenario comprising of multiband 5G NR at $3 \mathrm{GHz}(20 \mathrm{MHz})$ and $10 \mathrm{GHz}(20 \mathrm{MHz})$, which was mentioned in our prior work [54], was used to validate this technique, but no DPD was used. As an upgrade to this architecture, a multiband DPD block has been added to this arrangement to improve the speed of this link. A dual drive Mach Zehnder Modulator (MZM) operating with two separate RF-driven signals, and a $1310 \mathrm{~nm}$ DFB laser is used in the system depicted in Figure 11. The Vector Signal Generator (VSG) labelled as VSG1 transmits RF1, a 5G NR waveform at $10 \mathrm{GHz}$, while the 5G transceiver transmits RF2, a $3 \mathrm{GHz}$ flexible waveform signal. The DPD process can be broken down into three stages.

At first, these signals undergo up-conversion one by one at their respective carrier frequencies of 3 and $20 \mathrm{GHz}$, respectively, before passing them through $10 \mathrm{~km}$ of Standard Single-Mode Fiber (SSMF) and receiving them with a photodetector $(0.71 \mathrm{~A} / \mathrm{W})$ that receives the signal and converts it back to the electrical domain. An amplification step is introduced since the multiband needs to be separated independently. The $10 \mathrm{GHz}$ and $3 \mathrm{GHz}$ signals are separated using a diplexer (DPX). After that, the signals are sent to several vector signal analyzers (VSA). For performance evaluation, each VSA output is passed to the post-processing block. This phase is done without DPD, which means the output is evaluated without going through the DPD process.

The DPD method represented in Figure 4 is used in the second phase, referred to as the DPD training phase, and training is used until the error converges.

To put it another way, DPD validates the theory behind inverting the amplitude and phase responses obtained at electrical amplifiers EA1 and EA2. CNN methods can be used depending on the user's needs and comparative requirements.

We use the PRS (positioning reference signal) given in the 5G NR architecture to accomplish synchronization for the received waveforms (both, input and output). PRS is assumed to have a bandwidth of $20 \mathrm{MHz} / 106$ resource blocks. In the time domain, the received and output reference broadcast signals are correlated, and the PDP (power delay profile) is processed through the maximum block to determine the strongest path of arrival.

In the third step, the pre-distorted baseband signals are fed into the DPD block, where they are upconverted to their carrier frequency by their respective VSGs before being fed onto the optical connection. The signal obtained at the photodiode is then transmitted through a diplexer DPX to isolate the various multi-bands before being sent to the DPD training step. We flip the switches in the opposite direction during the DPD validation step. The evaluation for 5G NR frames is completed by predistortion and then transferring the frames to the VSG. Because the nonlinearities of the RoF link slowly change due to thermal 
impacts and component ageing, we conclude that real-time operation in the adaptation is unnecessary. Table 2 summarizes the parameters employed, which have previously been used in [1,2] and other state-of-the-art [54,55] studies.

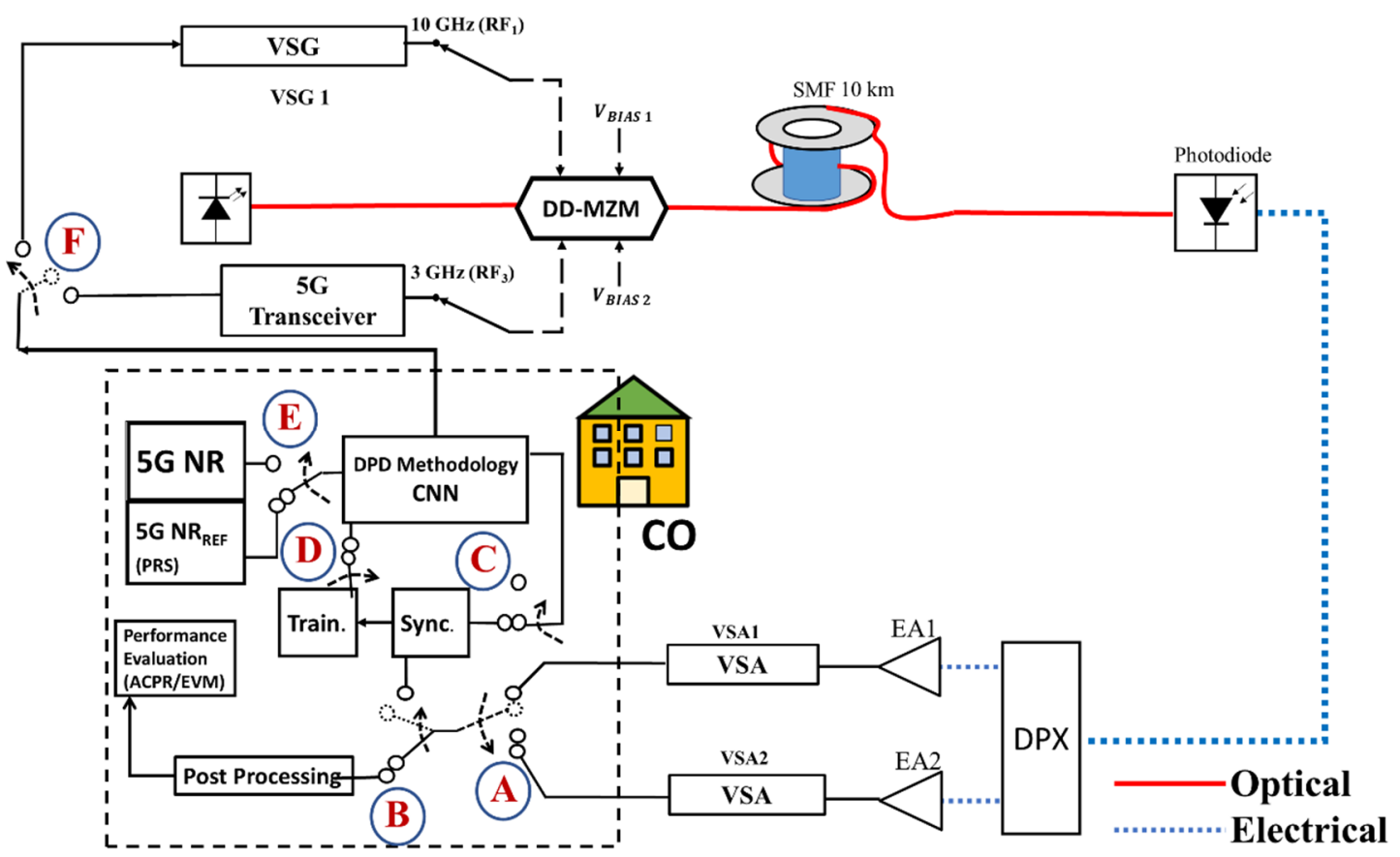

Figure 4. Experimental block diagram for analog multiband 5G NR system. The functions: A: Choose between VSA 1 and VSA 2. B: Choose between a performance post-processing block and a synchronisation block. C: Synch is connected. The synchronisation block is followed by training. D: DPD disables the training DPD disables the training DPD disables the training DPD disables the training DPD disable E: Time synchronisation (TS) technique is required. F: Required for validating DPD inputs before sending them to the VSGs. For the DPD training CNN has been chosen.

Table 2. Optical Link Parameters.

\begin{tabular}{cc}
\hline Parameters & Values \\
\hline 5G NR Waveforms & $f_{c}=3$ and $10 \mathrm{GHz}$ \\
& $\begin{array}{c}\text { Flexible O/G/F OFDM waveform } \\
\text { Modulation }=256 \text { QAM }\end{array}$ \\
\hline Laser Diode & $\lambda=1310 \mathrm{~nm}$ \\
& DD-MZM \\
Fiber & SSMF \\
& Optical Fibre Dispersion $=16 \frac{\mathrm{ps}}{\mathrm{nmkm}}$ \\
Length $=10 \mathrm{~km}$ \\
Photoreceiver & Attenuation $=0.44 \frac{\mathrm{dB}}{\mathrm{km}}$ \\
\hline & $\mathcal{R}=0.69 \mathrm{~A} / \mathrm{W}$ \\
\hline
\end{tabular}

Table 3 displays the CNN parameters that result in NN performance that is optimised. Table 3 lists the parameters that define the architecture of the intended Convolutional Neural Network. The parameter selection is done by a trial-and-error test [1]. The table's final section assesses the NN's complexity by computing its expressions in terms of its coefficients. 
Table 3. CNN parameters.

\begin{tabular}{cc}
\hline Framework & Parameters \\
\hline Optimiser & ADAM \\
ReLu \\
Type of activations function & Softmax \\
O/P layer type function & Mean Square Error (MSE) \\
Loss type & 15 \\
Hidden Layers $N$ & 20 \\
Neurons per layer $K$ & L2 \\
Regularization method & 0.001 \\
Regularization factor & 100 \\
Number of epochs & 0.4 \\
Validation split & 0.01 \\
Learning rate & $16,32,64,128,256,512,1024$ \\
Batch size & 300,000 \\
Training specimens & 300,000 \\
Testing specimens & \\
\hline Involution & 5986 \\
\hline
\end{tabular}

\section{Results and Discussion}

The results for the experimental setup outlined in the previous part are discussed in this section. The Mean Square Error (MSE) is one of the methods for estimating the accuracy of coefficient estimation for various architectures. From our previous work, GMP and Magnitude Selective Affine (MSA) DPD methods results are used to compare with our proposed CNN method. When no DPD is used, the MSE is $27 \mathrm{~dB}$, while GMP has $30 \mathrm{~dB}$. For Canonical Piecewise Linearization (CPWL) and MSA, the value drops to $35 \mathrm{~dB}$, whereas CNN has an MSE of $39 \mathrm{~dB}$.

The proposed approach is compared and reported in the form of Error Vector Magnitude (EVM). From our recent work [2], we just use GMP methods as a baseline architecture to compare with our proposed CNN method.

\section{Error Vector Magnitude}

The most common performance indicator utilised in 3GPP for this research item's performance evaluation is Error Vector Magnitude. The difference between the symbol's demodulated 'anticipated' value and the demodulated received symbols 'real' value is determined by EVM. EVM can be expressed as [5,55]:

$$
\operatorname{EVM}(\%)=\sqrt{\frac{\frac{1}{M} \sum_{m=1}^{M}\left|S_{m}-S_{0, m}\right|^{2}}{\frac{1}{M} \sum_{m=1}^{M}\left|S_{m}\right|^{2}}}
$$

where $M$ is the number of constellation symbols, $S_{m}$ denotes the constellation's real symbol associated with the symbol " $m$ " and $S_{0, m}$ is the real symbol associated with $S_{m}$. The EVM limit for 3GPP using 256 QAM is 3.5\% [56].

In Figure 5a, the Error Vector Magnitude EVM is represented for flexible waveforms compared for the case when there is no DPD improvement procedure employed when GMP DPD method is employed, and the CNN DPD method is utilized. It is observable that the CNN DPD method results in a better reduction as compared to GMP for all the flexible waveform architectures. Similarly in Figure 5b, it can be seen that when the RF input power is varied, the reduction in EVM due to the proposed CNN method is much better as compared to GMP method. For a high RF input power of $5 \mathrm{dBm}$, DPD CNN reduces EVM to approximately 2\% while GMP has EVM around 5\% so EVM reduction is about $9 \%$ with the CNN method bringing the performance within the limits set by 3GPP. 


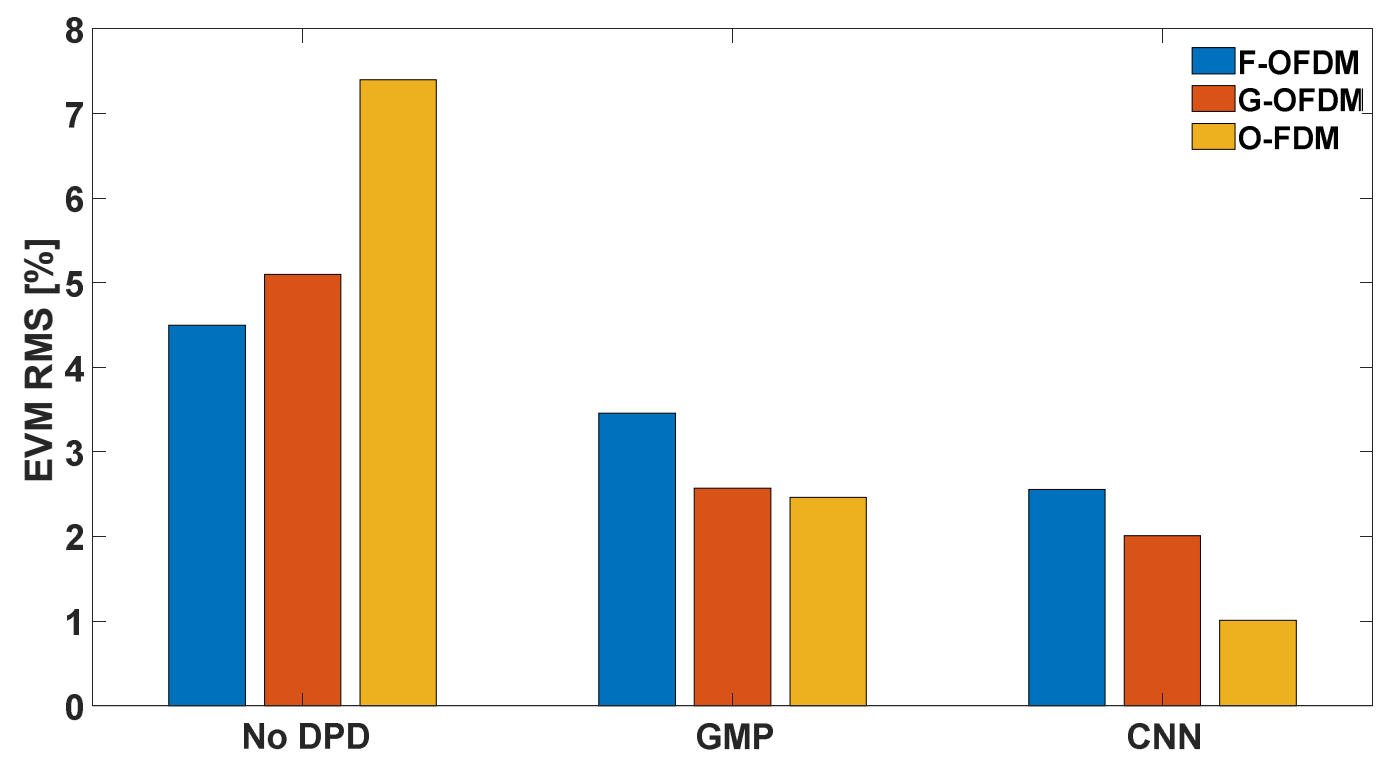

(a)

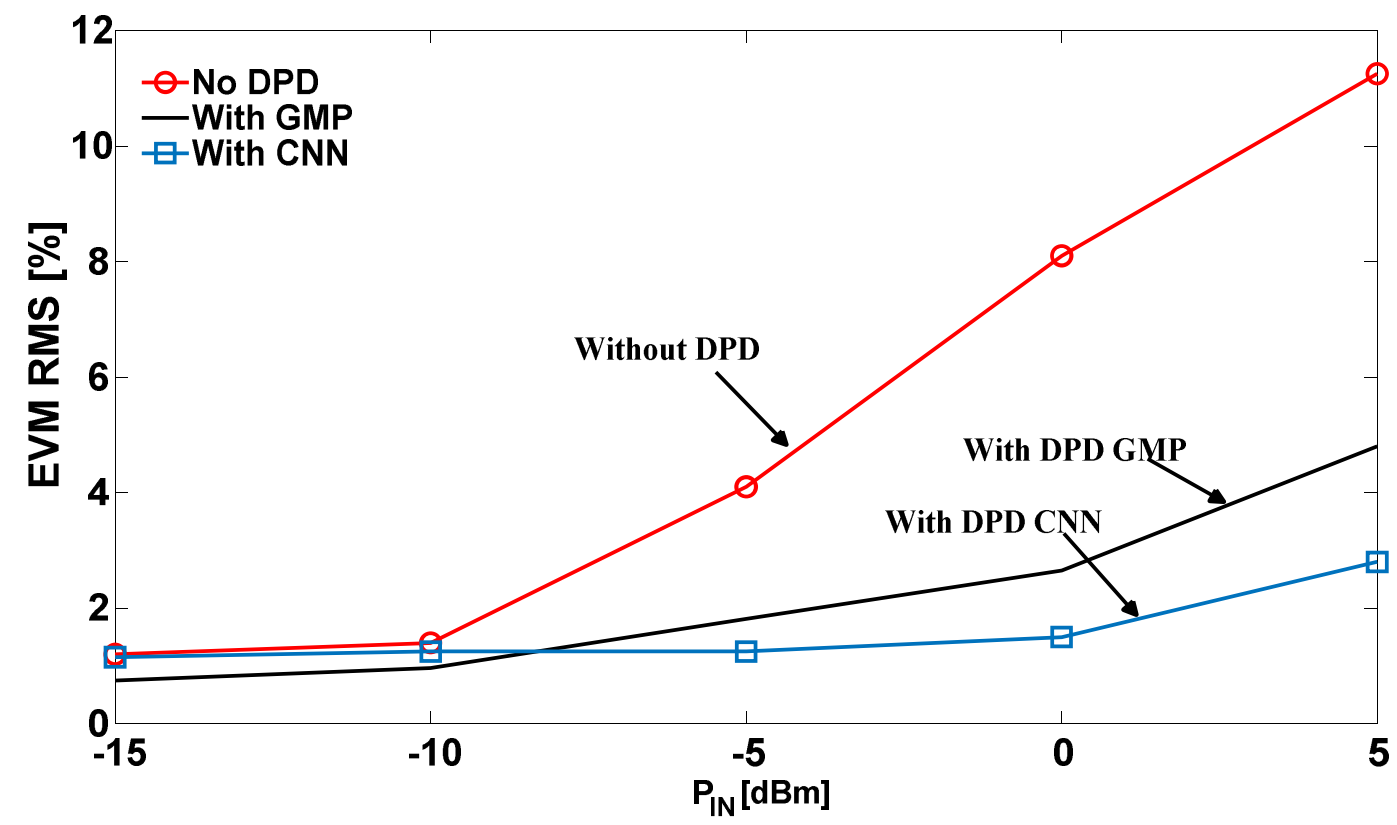

(b)

Figure 5. EVM performance comparison (a) for CNN and No DPD case for flexible 5G NR waveforms. (b) 5G NR performance DPD efficacy in terms of EVM for proposed CNN DPD method vs GMP method and without DPD for varying RF input power.

\section{Complexity Considerations}

By lowering complexity and attaining equivalent performance to the DNN approach outlined in prior work [2], CNN-DPD achieves a significant improvement. Table 4 depicts the difficulty of creating a DPD model, which is mostly determined by the number of actual multipliers necessary, as multipliers use the majority of the hardware resources. MSA DPD (220 multiplications) is significantly less complicated than CPWL, as seen in Table 4 . (880 multiplications). More complex versions can be constructed by increasing the memory depth $Q$ and nonlinearity order $K$ in the Volterra series. However, the computational 
complexity must be considered. This means that while choosing the DPD model and its complexity, a reasonable trade-off between complexity and performance may be made.

For a comprehensive review of CNN and Volterra techniques in the manner of complexity, Table 4 summarizes the complexities for the methods. CNN complexity is a challenging problem to solve, however as shown in this work, it can be reduced by employing a minimal number of $N$ and $K$.

Table 4. Comparisons of complexity.

\begin{tabular}{cccc}
\hline DPD & Coefficients & \# Coefficients & Arithmetic Operations \\
\hline GMP & $K_{c}\left(Q_{c}+1\right) R_{c}+K_{b}\left(Q_{b}+1\right) R_{b}+K_{a}\left(Q_{a}+1\right)$ & 84 & 244 \\
CPWL & $(4 M+1)(K+1) L$ & 260 & $(K+1)(14 M+2) L=880$ \\
MSA & $2(4 M+1)(K+1) L$ & 520 & $(14 M+2)(K+1)=220$ \\
CNN & $(N-1) K^{2}+(4+N) K+6$ & 5986 & $4 K+4+(N-1) K^{2}=5684$ \\
\hline
\end{tabular}

Indeed, with the higher modulation format and higher bandwidth similar to multiple LTE carriers or 5G new radio (NR) waveforms as discussed, they would lead to higher complexity of DPD operation due to stronger PAPR. Concomitantly, the elevation in bandwidth will lead to an overall increase in the base-band memory of the system model. Nevertheless, the evaluated models are still valid. However, higher values of the $\mathrm{Q}$ and $\mathrm{K}$ will be indispensable as compared to the considered case.

In Table 5, the values of MSE and EVM @ 5dBm are summarized for the proposed method.

Table 5. Results summary for EVM and MSE.

\begin{tabular}{ccc}
\hline Methodology & E.V.M (\%) & MSE (dB) \\
\hline No-Digital Pre-distortion & 11 & -27 \\
GMP Digital Pre-distortion & 5 & -30 \\
CNN Digital Pre-distortion & 2.1 & -39 \\
\hline
\end{tabular}

\section{Conclusions}

This paper describes the effective implementation of 5G NR multiband OFH utilising Convolutional Neural Networks to reduce RoF nonlinearities. To begin, a novel CNN approach was developed that not only has less complexity than conventional machine learning methods but also performs better with a $75 \%$ decrease in complexity overheads and multiplications. The theoretical foundations and pieces needed to design a Neural Network are also explained in the article. To a distance of $10 \mathrm{~km}, 5 \mathrm{G}$ New Radio multiband transmissions at $3 \mathrm{GHz}$ and $10 \mathrm{GHz}$ are used. The proposed CNN-DPD approach reduces EVM by $8 \%$ to $2.1 \%$ bringing the performance within 3GPP limits.

Funding: This research received no external funding.

Institutional Review Board Statement: Not Applicable.

Data Availability Statement: The data presented in this study are available on request from the corresponding author.

Conflicts of Interest: The author declare no conflict of interest.

\section{References}

1. Hadi, M.; Awais, M.; Raza, M.; Khurshid, K.; Jung, H. Neural Network DPD for Aggrandizing SM-VCSEL-SSMF-Based Radio over Fiber Link Performance. Photonics 2021, 8, 19. [CrossRef]

2. Hadi, M.U.; Awais, M.; Raza, M.; Ashraf, M.I.; Song, J. Experimental Demonstration and Performance Enhancement of 5G NR Multiband Radio over Fiber System Using Optimized Digital Predistortion. Appl. Sci. 2021, 11, 11624. [CrossRef]

3. Nanni, J.; Polleux, J.-L.; Algani, C.; Rusticelli, S.; Perini, F.; Tartarini, G. VCSEL-based radio-over-G652 fiber system for short/medium-range MFH solutions. J. Lightwave Technol. 2018, 36, 4430-4437. [CrossRef]

4. Khurshid, K.; Khan, A.A.; Siddiqui, H.; Rashid, I.; Hadi, M.U. Big Data Assisted CRAN Enabled 5G SON Architecture. J. ICT Res. Appl. 2019, 13, 93-106. [CrossRef] 
5. Nanni, J.; Baschieri, L.; Polleux, J.-L.; Tartarini, G.; Hadi, M.U. Effective Digital Pre-Distortion Loop for Front Hauls based on short- $\lambda$-VCSELs over pre-existent G-652 Infrastructures. In Proceedings of the 2021 International Topical Meeting on Microwave Photonics (MWP), Pisa, Italy, 15-17 November 2021; pp. 1-4. [CrossRef]

6. Aslam, N.; Xia, K.; Haider, M.T.; Hadi, M.U. Energy-Aware Adaptive Weighted Grid Clustering Algorithm for Renewable Wireless Sensor Networks. Future Internet 2017, 9, 54. [CrossRef]

7. Zhang, X.; Zhu, R.; Shen, D.; Liu, T. Linearization Technologies for Broadband Radio-Over-Fiber Transmission Systems. Photonics 2014, 1, 455-472. [CrossRef]

8. Li, H.; Bauwelinck, J.; Demeester, P.; Torfs, G.; Verplaetse, M.; Verbist, J.; Van Kerrebrouck, J.; Breyne, L.; Wu, C.-Y.; Bogaert, L.; et al. Real-Time 100-GS/s Sigma-Delta Modulator for All-Digital Radio-Over-Fiber Transmission. J. Lightwave Technol. 2020, 38, 386-393. [CrossRef]

9. Hadi, M.U.; Jung, H.; Ghaffar, S.; Traverso, P.A.; Tartarini, G. Optimized digital radio over fiber system for medium range communication. Opt. Commun. 2019, 443, 177-185. [CrossRef]

10. Wang, J.; Jia, Z.; Campos, L.A.; Knittle, C.; Jia, S. Delta-Sigma Modulation for Next Generation Fronthaul Interface. J. Lightwave Technol. 2018, 37, 2838-2850. [CrossRef]

11. Nanni, J.; Giovannini, A.; Hadi, M.U.; Lenzi, E.; Rusticelli, S.; Wayth, R.; Perini, F.; Monari, J.; Tartarini, G. Controlling RayleighBackscattering-Induced Distortion in Radio Over Fiber Systems for Radioastronomic Applications. J. Lightwave Technol. 2020, 38, 5393-5405. [CrossRef]

12. Van Kerrebrouck, J.; Breyne, L.; Li, H.; Bauwelinck, J.; Torfs, G.; Demeester, P.; Bohn, T. Real-Time All-Digital Radioover-Fiber LTE Transmission. In Proceedings of the 2017 Advances in Wireless and Optical Communications (RTUWO), Riga, Latvia, 2-3 November 2017; pp. 83-86.

13. Li, H.; Hajipour, J.; Attar, A.; Leung, V.C.M. Efficient HetNet implementation using broadband wireless access with fiber-connected massively distributed antennas architecture. IEEE Wirel. Commun. 2011, 18, 72-78. [CrossRef]

14. Wang, D.; Zhang, M.; Fu, M.; Cai, Z.; Li, Z.; Han, H.; Cui, Y.; Luo, B. Nonlinearity mitigation using a machine learning detector based on k-nearest neighbors. IEEE Photonics Technol. Lett. 2016, 28, 2102-2105. [CrossRef]

15. Huang, Y.; Chen, Y.; Yu, J. Nonlinearity mitigation of RoF signal using machine learning based classifier. In Proceedings of the Asia Communications and Photonics Conference, Guangzhou, China, 10-13 November 2017; p. Su2A.28.

16. Cui, Y.; Zhang, M.; Wang, D.; Liu, S.; Li, Z.; Chang, G.K. Bit-based support vector machine nonlinear detector for millimeter-wave radio-over-fiber mobile fronthaul systems. Opt. Express 2017, 25, 26186-26197. [CrossRef] [PubMed]

17. Li, D.; Yu, S.; Jiang, T.; Han, Y.; Gu, W. An M-ary SVM-based detection for 16-QAM RoF system with data-dependent cross modulation distortion. In Proceedings of the Asia Communications and Photonics Conference, Hong Kong, 19-23 November 2015; p. ASu3J.4.

18. Gonzalez, N.G.; Zibar, D.; Caballero, A.; Monroy, I.T. Experimental 2.5-Gb/s QPSK WDM Phase-Modulated Radio-Over-Fiber Link with Digital Demodulation by a KK-Means Algorithm. IEEE Photonics Technol. Lett. 2010, 22, 335-337. [CrossRef]

19. Fernandez, E.A.; Torres, J.J.G.; Soto, A.M.C.; Gonzalez, N.G. Radio-over-fiber signal demodulation in the presence of non-Gaussian distortions based on subregion constellation processing. Opt. Fiber Technol. 2019, 53, 102062. [CrossRef]

20. Khurshid, K.; Khan, A.A.; Siddiqui, M.H.; Hadi, M.U.; Rashid, I.; Imran, M. Optimality of Linear MIMO Detection for 5G Systems via 1-Opt Local Search. J. Electr. Eng. Technol. 2021, 16, 1099-1108. [CrossRef]

21. Hadi, M.U.; Mittal, I. On the Use of SVR based Machine Learning Method for Nonlinearities Mitigation in Short Range Fronthaul Links. In Proceedings of the 2021 10th IEEE International Conference on Communication Systems and Network Technologies (CSNT), Bhopal, India, 18-19 June 2021; pp. 628-631.

22. Hadi, M.U.; Basit, A. Machine Learning for Performance Enhancement in Fronthaul Links for IOT Applications. In Proceedings of the 2021 International Conference on Digital Futures and Transformative Technologies (ICoDT2), Islamabad, Pakistan, 20-21 May 2021; pp. 1-5.

23. Lee, J.; He, J.; Wang, Y.; Fang, C.; Wang, K. Experimental demonstration of millimeter-wave radio-over-fiber system with convolutional neural network and binary convolutional neural network (BCNN). arXiv 2020, arXiv:2001.02018. Available online: https:/ / arxiv.org/abs/2001.02018 (accessed on 5 December 2021).

24. Lee, J.; He, J.; Wang, K. Neural networks and FPGA hardware accelerators for millimeter-wave radio-over-fiber systems. In Proceedings of the International Conference on Transparent Optical Networks, Bari, Italy, 19-23 July 2020; p. Mo.D1.5.

25. Liu, S.; Wang, X.; Zhang, W.; Shen, G.; Tian, H. An Adaptive Activated ANN Equalizer Applied in Millimeter-Wave RoF Transmission System. IEEE Photonics Technol. Lett. 2017, 29, 1935-1938. [CrossRef]

26. Liu, S.; Xu, M.; Wang, J.; Lu, F.; Zhang, W.; Tian, H.; Chang, G.-K. A Multilevel Artificial Neural Network Nonlinear Equalizer for Millimeter-Wave Mobile Fronthaul Systems. J. Lightwave Technol. 2017, 35, 4406-4417. [CrossRef]

27. Liu, S.; Alfadhli, Y.M.; Shen, S.; Tian, H.; Chang, G.K. Mitigation of multi-user access impairments in 5G A-RoF-based mobilefronthaul utilizing machine learning for an artificial neural network nonlinear equalizer. In Proceedings of the Optical Fiber Communication Conference, San Diego, CA, USA, 11-15 March 2018.

28. Liu, E.; Yu, Z.; Yin, C.; Xu, K. Nonlinear distortions compensation based on artificial neural networks in wideband and multi-carrier systems. IEEE J. Quantum Electron. 2019, 55, 800305. [CrossRef]

29. Liu, J.; Zou, X.; Bai, W. Performance enhancement of UFMC based radio over fiber system using ANN equalizer. In Proceedings of the Asia Communications and Photonics Conference, Hangzhou, China, 26-29 October 2018. 
30. Safari, L.; Baghersalimi, G.; Karami, A.; Kiani, A. On the Equalization of an OFDM-Based Radio-over-Fiber System Using Neural Networks. Radioengineering 2017, 26, 162-169. [CrossRef]

31. Zhou, Q.; Lu, F.; Xu, M.; Peng, P.-C.; Liu, S.; Shen, S.; Zhang, R.; Yao, S.; Finkelstein, J.; Chang, G.-K. Enhanced Multi-Level Signal Recovery in Mobile Fronthaul Network Using DNN Decoder. IEEE Photonics Technol. Lett. 2018, 30, 1511-1514. [CrossRef]

32. Xu, T.; Shevchenko, A.N.; Zhang, Y.; Jin, C.; Zhao, J.; Liu, T. Information rates in Kerr non-linearity limited optical fiber communication systems. Opt. Express 2021, 29, 17428-17439. [CrossRef] [PubMed]

33. Draa, M.N.; Hastings, A.S.; Williams, K.J. Comparison of photodiode nonlinearity measurement systems. Opt. Express 2011, 19, 12635-12645. [CrossRef] [PubMed]

34. Chen, Z.; Yan, L.; Pan, W.; Luo, B.; Zou, X.; Guo, Y.; Jiang, H.; Zhou, T. SFDR enhancement in analog photonic links by simultaneous compensation for dispersion and nonlinearity. OSA Optics Expr. 2013, 21, 20999-21009. [CrossRef]

35. Haas, B.M.; Murphy, T. A Simple, Linearized, Phase-Modulated Analog Optical Transmission System. IEEE Photonics Technol. Lett. 2007, 19, 729-731. [CrossRef]

36. Zhu, R.; Zhang, X. Linearization of Radio-Over-Fiber Systems by Using Two Lasers with Different Wavelengths. In Proceedings of the 2014 IEEE MTT-S International Microwave Symposium (IMS), Tampa, FL, USA, 1-6 June 2014.

37. Ghannouchi, F.; Younes, M.; Rawat, M. Distortion and impairments mitigation and compensation of single- and multi-band wireless transmitters. IET Microw. Antennas Propag. 2013, 7, 518-534. [CrossRef]

38. Duan, R.; Xu, K.; Dai, J.; Cui, Y.; Wu, J.; Li, Y.; Dai, Y.; Li, J. Linearity improvement based on digital signal processing in intensity-modulated analog optical links incorporating photonic frequency down conversion. In Proceedings of the Optical Fiber Communication Conference and Exposition, Los Angeles, CA, USA, 6-8 March 2012.

39. Pei, Y.; Xu, K.; Li, J.; Zhang, A.; Dai, Y.; Ji, Y.; Lin, J. Complexity-reduced digital predistortion for subcarrier multiplexed radio over fiber systems transmitting sparse multi-band RF signals. Opt. Express 2013, 21, 3708-3714. [CrossRef]

40. Vieira, L.; Gomes, N.J.; Nkansah, A.; van Dijk, F. Behavioral modeling of radio-overfiber links using memory polynomials. In Proceedings of the 2010 IEEE Topical Meeting on Microwave Photonics (MWP), Montreal, QC, Canada, 5-9 October 2010; pp. 85-88.

41. Hekkala, A.; Hiivala, M.; Lasanen, M.; Perttu, J.; Vieira, L.C.; Gomes, N.J.; Nkansah, A. Predistortion of Radio Over Fiber Links: Algorithms, Implementation, and Measurements. IEEE Trans. Circuits Syst. I Regul. Pap. 2011, 59, 664-672. [CrossRef]

42. Fuochi, F.; Hadi, M.U.; Nanni, J.; Traverso, P.A.; Tartarini, G. Digital predistortion technique for the compensation of nonlinear effects in radio over fiber links. In Proceedings of the 2016 IEEE 2nd International Forum on Research and Technologies for Society and Industry Leveraging a Better Tomorrow (RTSI), Bologna, Italy, 7-9 September 2016; pp. 1-6.

43. Vieira, L.C.; Gomes, N.J.; Nkansah, A. An experimental study on digital predistortion for radio-over-fiber links. In Proceedings of the Asia Communications and Photonics Conference and Exhibition, Shanghai, China, 8-12 December 2010; pp. 126-127. [CrossRef]

44. Hekkala, A.; Lasanen, M.; Vieira, L.C.; Gomes, N.J.; Nkansah, A. Architectures for Joint Compensation of RoF and PA with Nonideal Feedback. In Proceedings of the 2010 IEEE 71st Vehicular Technology Conference, Taipei, Taiwan, 16-19 May 2010; pp. 1-5.

45. Mateo, C.; Carro, P.L.; Garcia-Ducar, P.; De Mingo, J.; Salinas, I. Experimental evaluation of the feedback loop effects in digital predistortion of a radio-over-fiber system. In Proceedings of the 2017 Ninth International Conference on Ubiquitous and Future Networks (ICUFN), Milan, Italy, 4-7 July 2017; pp. 1039-1041.

46. Mateo, C.; Clemente, J.; Garcia-Ducar, P.; Carro, P.L.; de Mingo, J.; Salinas, I. Digital predistortion of a full-duplex Radio-over-Fiber mobile fronthaul link with feedback loop. In Proceedings of the 2017 13th International Wireless Communications and Mobile Computing Conference (IWCMC), Valencia, Spain, 26-30 June 2017; pp. 1425-1430.

47. Mateo, C.; Carro, P.L.; García-Dúcar, P.; De Mingo, J.; Salinas, I. Minimization of Feedback Loop Distortions in Digital Predistortion of a Radio-Over-Fiber System with Optimization Algorithms. IEEE Photonics J. 2017, 9, 7904414. [CrossRef]

48. Roselli, L.; Borgioni, V.; Zepparelli, F.; Ambrosi, F.; Comez, M.; Faccin, P.; Casini, A. Analog laser predistortion for multiservice radio-over-fiber systems. J. Lightwave Technol. 2003, 21, 1211-1223. [CrossRef]

49. Hadi, M.U.; Jung, H.; Traverso, P.A.; Tartarini, G. Experimental evaluation of real-time sigma-delta radio over fiber system for fronthaul applications. Int. J. Microw. Wirel. Technol. 2020, 13, 756-765. [CrossRef]

50. Hraimel, B.; Zhang, X. Low-cost broadband predistortion-linearized single drive X-cut Mach-Zehnder modulator for radio-overfiber systems. Photonics Technol. Lett. 2012, 24, 1571-1573. [CrossRef]

51. Habib, U.; Aighobahi, A.E.; Quinlan, T.; Walker, S.D.; Gomes, N.J. Analog Radio-Over-Fiber Supported Increased RAU Spacing for $60 \mathrm{GHz}$ Distributed MIMO Employing Spatial Diversity and Multiplexing. J. Lightwave Technol. 2018, 36, 4354-4360. [CrossRef]

52. Jiang, D.; Liu, G. An Overview of 5G Requirements. In 5G Mobile Communications; Xiang, W., Zheng, K., Shen, X., Eds.; Springer: Cham, Switzerland, 2017; pp. 3-26.

53. He, J.; Lee, J.; Kandeepan, S.; Wang, K. Machine Learning Techniques in Radio-over-Fiber Systems and Networks. Photonics 2020, 7, 105. [CrossRef]

54. Yang, Y.-S.; Huang, W.-C.; Li, C.-P.; Li, H.-J.; Stüber, G.L. A Low-Complexity Transceiver Structure with Multiple CFOs Compensation for OFDM-Based Coordinated MultiPoint Systems. IEEE Trans. Commun. 2015, 63, 2658-2670. [CrossRef] 
55. Aslam, N.; Xia, K.; Hadi, M.U. Optimal Wireless Charging Inclusive of Intellectual Routing Based on SARSA Learning in Renewable Wireless Sensor Networks. IEEE Sens. J. 2019, 19, 8340-8351. [CrossRef]

56. LTE. Evolved Universal Terrestrial Radio Access (E-UTRA); ETSI, TS 136104 V8.2.0; Base Station (BS) Radio Transmission and Reception; European Telecommunications Standards Institute: Valbonne, France, 2018. 